\title{
O USO DO TUBO COLÔNICO BIPEDICULADO MICROCIRÚRGICO NA RECONSTRUÇÃO DO ESÔFAGO
}

\author{
THE USE OF MICROSURGICAL BIPEDICLE COLONIC TUBE IN ESOPHAGEAL \\ RECONSTRUCTION
}

\section{INTRODUÇÃO}

Em algumas situações, a reconstrução esofágica após esofagectomia não pode ser realizada com tubo gástrico. Por exemplo, nos tumores da cárdia com invasão para o estômago proximal, tumores no terço distal do esôfago que invadam a pequena curvatura do estômago e em casos que foram realizadas cirurgias prévias com ressecções gástricas parciais ${ }^{1}$. Nestas condições, preconiza-se a utilização do cólon.

Como complicações desta cirurgia podemos citar o alto índice de fistulas das anastomoses na região do esôfago cervical, assim como estenoses por isquemia ${ }^{2}$. No caso apresentado, com o objetivo de melhorar a perfusão e evitar tais complicações, utilizamos um segundo pedículo vascular na parte distal deste tubo colônico através da anastomose microcirúrgica, funcionando como um "super-charge".

\section{RELATO DO CASO}

Paciente de 52 anos, masculino, negro, com carcinoma epidermóide do terço médio do esôfago, submetido a esofagectomia total e reconstrução com transposição gástrica. No quarto dia de pós-operatório apresentou necrose do segmento gástrico proximal tendo sido realizada a retirada do estômago e confecção de esofagostomia e jejunostomia. Após quatro meses, foi submetido a nova reconstrução com

\section{Mário Sérgio Lomba Galvão, TCBC-RJ ${ }^{1}$ \\ Carlos Eduardo Pinto, TCBC-RJ ${ }^{2}$ Juliano Carlos Sbalchiero ${ }^{3}$}

transposição colônica bipediculada, com anastomose microcirúrgica. Foi utilizado segmento colônico de 40 $\mathrm{cm}$ (transverso e descendente) com ligadura da artéria mesentérica inferior, mantendo-se a vascularização pela artéria cólica média e drenagem com as veias comitantes (Figura 1). O tubo colônico foi posicionado no sentido anisoperistáltico retroesternal e a anastomose microcirúrgica ("super-charge") foi realizada entre os vasos hemorroidários superiores e artéria e veia cervical transversa de forma término-terminal, utilizando fios oito e nove zeros, respectivamente (Figura 2). A evolução pós-operatória transcorreu sem anormalidades. Após exame contrastado no $10^{\circ}$ dia pós-operatório, afastou-se a possibilidade de fístula e o paciente apresentou boa reabilitação funcional e deglutição normal, com recuperação do estado nutricional.

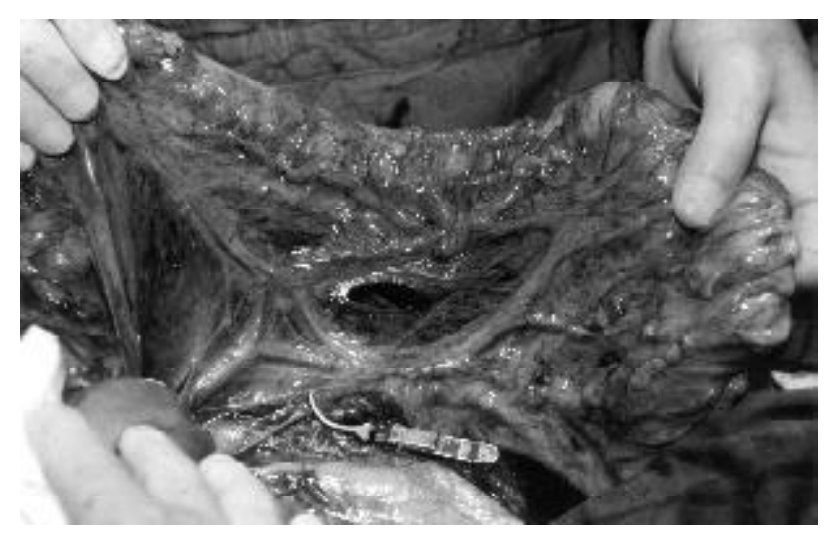

Figura 1 - Isolamento do segmento colônico a ser transposto.

1. Fundador do Serviço de Microcirurgia do INCa

2. Staff do Serviço de Cirurgia Oncológica do INCa

3. Staff do Serviço Cirurgia Plástica e Microcirurgia do INCa

Recebido em: 19/11/2001

Aceito para publicação em: 18/03/2003

Trabalho realizado no Instituto Nacional de Câncer - INCa - RJ 


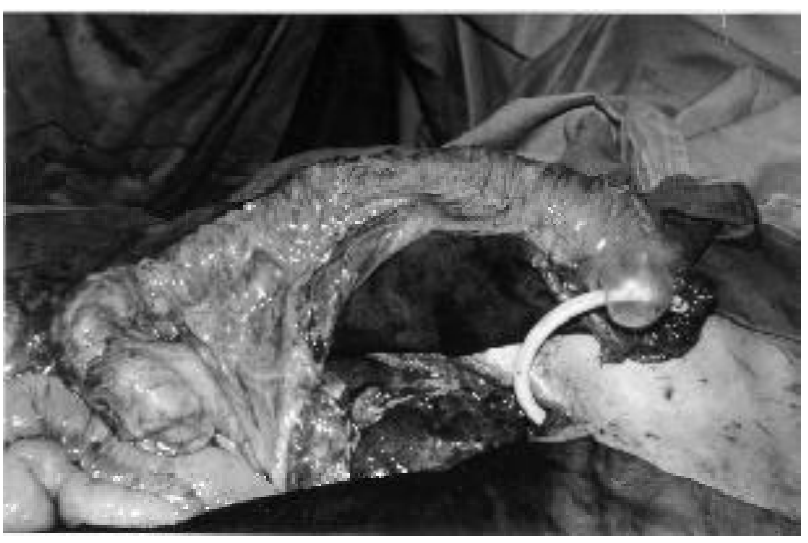

Figura 2 - Transposição do segmento colônico e anastomose proximal.

\section{DISCUSSÃO}

O restabelecimento do trânsito alimentar nas esofagectomias é de suma importância para o suporte nutricional e a preservação da qualidade de vida. Dentre as complicações que ocorrem nas reconstruções de esôfago, talvez a que mais influencie neste aspecto seja a disfagia, cujas causas podem ser a estenose anastomótica, a recorrência tumoral e a estenose péptica no esôfago remanescente ${ }^{3}$.

Uma causa rara de disfagia é a estenose isquêmica, um diagnóstico de exclusão em relação às etiologias previamente mencionadas. Nestes casos, são descritos dois tipos. O primeiro ocorre durante a cirurgia, quando são observados sinais leves de isquemia, como cianose da alça e o aumento da peristalse. Este dano isquêmico pode ser reversível ou vir a causar estenose dentro de poucas semanas. No segundo tipo, a estenose pode se desenvolver meses ou anos mais tarde por angulação dos vasos nutrientes ou fibrose perivascular, ou ainda por aterosclerose progressiva ${ }^{4}$.

Durante o período de isquemia as diferentes camadas das vísceras ocas sofrem danos diversos. Primeiro, a camada epitelial suprida por endocapilares é envolvida, seguindo-se da camada subepitelial. Com isso, a permeabilidade das membranas celulares aumenta $\mathrm{e}$ o edema se instala, levando à morte celular e conseqüentemente ao rompimento da mucosa. Se as camadas profundas não são envolvidas, existe a possibilidade de regeneração completa, porém, se atingir a camada muscular, ela sofrerá um processo de fibrose que culminará com a estenose. Para que haja o processo regenerativo, normalmente necessitase de uma a três semanas, dependendo da gravidade da lesão. Na necrose de espessura total, não existe chance de regeneração ${ }^{5}$.

A isquemia tem uma repercussão importante quanto mais distal estiver o segmento de alça em relação ao pedículo. Uma vez restaurada a perfusão, e as alterações microscópicas já se instalaram, a reperfusão acarreta produção de radicais de superóxido e lesão celular ${ }^{4}$. Outros fatores que podem influenciar negativamente a perfusão são algumas drogas (digital, atropina, nicotina) e ainda a microangiopatia do diabetes ou a manifestação intestinal da aterosclerose.

A microcirurgia possibilita um suprimento vascular adicional na porção distal da alça que é mais suscetível à isquemia e permite uma perfusão adequada desde os momentos iniciais da reconstrução, evitando, portanto, a instalação das alterações isquêmicas citadas até que os mecanismos fisiológicos de adaptação permitam uma perfusão adequada do segmento distal pelo pedículo proximal.

\begin{abstract}
In some cases of esophageal reconstruction, it is not possible to use the gastric tube. In those cases, the second option is the reconstruction with a colonic segment. In the present paper, the authors present the use of microsurgical technique to improve vascular supply in esophageal reconstructions using the colon. Therefore, the transposed segment becomes perfused by two vascular pedicles: a proximal one and a distal one. The authors describe a case of 52 years-old patient, suffering of middle third esophagus carcinoma, who underwent a primarily esophageal resection with an unsuccessful reconstruction using gastric transposition. A new reconstruction was proposed using a bipedicle microcirurgical colonic tube, four months later. The post operative was uneventful with rehabilitation of swallowing and satisfactory recovery of nutritional state.
\end{abstract}

Key Words: Esophagus; Esophageal neoplasms; Carcinoma; Reconstruction; Microsurgery; Postoperative complications; Colon; Rehabilitation. 


\section{AGRADECIMENTOS:}

Os autores agradecem a Paulo Roberto Leal, Chefe do Serviço Cirurgia Plástica e Microcirurgia do INCA e a Marcela Caetano Cammarota, Médica Residente do Serviço Cirurgia Plástica e Microcirurgia do INCA.

\section{REFERÊNCIAS}

1. Coleman JJ - Reconstruction of the pharynx and cervical esophagus. Semin Surg Oncol, 1995, 11(3): 208220.

2. Robinson DW, McLeod A - Microvascular free jejunum transfer. Br J Plast Surg, 1982, 35(3): 258-267.

3. Surkin MI, Lawson W, Biller HF - Analysis of the methods of pharyngoesophageal reconstruction. Head Neck Surg, 1984, 6(5): 953-970.
4. Ashizawa I, Nishihira T, Kasai M, et al. - Improvement of circulation in pedicled intestinal grafts: hemodynamics of the intestine after preparation of a sacrificial colonic graft. J Am Coll Surg, 1997, 184(4): 346-352.

5. Nicks R - Colonic replacement of the oesophagus. Some observations on infarction and wound leakage. Br J Surg, 1967, 54(2): 124-128.

Endereço para correspondência:

Dr. Paulo Roberto Leal Instituto Nacional de Câncer Praça da Cruz Vermelha, ${ }^{\circ} 23$ Secretaria da Cirurgia Plástica $-8^{\circ}$ andar Cep: 20230-130 - Rio de Janeiro - RJ Tel: (21) 2506-6087

e-mail: prl@rio.com.br 